\title{
Santo. Les explorateurs de l'île planète de Vincent Tardieu et Lise Barnéoud
}

\section{Gilles Bounoure}

\section{(2) OpenEdition \\ 1 Journals}

Édition électronique

URL : http://journals.openedition.org/jso/1982

DOI : $10.4000 /$ jso. 1982

ISSN : 1760-7256

Éditeur

Société des océanistes

\section{Édition imprimée}

Date de publication : 15 décembre 2008

Pagination : 336-337

ISBN : 978-2-85430-012-3

ISSN : 0300-953x

\section{Référence électronique}

Gilles Bounoure, "Santo. Les explorateurs de lîle planète de Vincent Tardieu et Lise Barnéoud », Journal de la Société des Océanistes [En ligne], 126-127 | Année 2008, mis en ligne le 01 décembre 2008, consulté le 25 septembre 2020. URL : http://journals.openedition.org/jso/1982 ; DOI : https://doi.org/ 10.4000/jso.1982

Ce document a été généré automatiquement le 25 septembre 2020.

(c) Tous droits réservés 


\title{
Santo. Les explorateurs de l'île planète de Vincent Tardieu et Lise Barnéoud
}

\author{
Gilles Bounoure
}

\section{RÉFÉRENCE}

Tardieu Vincent et Lise Barnéoud, 2007. Santo. Les explorateurs de l'île planète. Préface de Nicolas Hulot, postface de Philippe Bouchet, Hervé Le Guyader et Olivier Pascal.

Paris, Belin, 288 p., cartes, plus de 350 ill. couleur.

1 Parmi les nombreuses «exploitations médiatiques» auxquelles a donné lieu l'expédition menée conjointement à Santo en 2006 par le Muséum national d'histoire naturelle de Paris, l'Institut de recherche pour le développement et l'association ProNatura international, cet ouvrage "grand public» se distingue par l'importance des contributions obtenues des scientifiques ayant pris part à cette vaste entreprise d'inventaire du " patrimoine naturel ». À côté de photographies spectaculaires et de nombreux commentaires et interviews, ils ont aussi livré aux deux auteurs, journalistes scientifiques, d'abondantes données techniques et des mises en perspectives qui font de l'ouvrage non seulement un livre intéressant et aisé à lire, mais un travail de vulgarisation de bon niveau, que délimitent assez bien le nom et le renom des signataires de la préface et de la postface. Surtout, cette publication semble la première à offrir, dans des délais relativement rapides, une vue d'ensemble de cette expédition récente, de ses enjeux et de ses premiers résultats.

2 Diverses bévues d'ordre culturel et historique déparent un peu la qualité «scientifique » du propos. Deux objets des îles de la Société (p. 41 : pectoral de deuil et p. 42 : herminette) résument plus que mal la civilisation matérielle ancienne de Santo ou même du Vanuatu. Censée attester l'importance locale du kava, la gravure figurant, selon sa légende, « l'assemblée des hommes et de la chefferie de l'île des Amis (actuelles îles Tonga) sous le nakamal » (sic: p. 155) semble de même prêter identité d'usages et de lexique à des sociétés traditionnelles du Pacifique très éloignées (voir aussi pp. 
158-159), l'ouvrage étant pourtant vendu sous une bande le célébrant comme un « éloge de la biodiversité »! Trop souvent erronées, les références des gravures reproduites peuvent même aller contre l'évidence : deux planches de la première édition française du deuxième voyage de Cook (Paris, Hôtel de Thou, 1778) sont ainsi présentées : "Ces superbes gravures, réalisées par l'artiste Bernard Direx... » (pp. 36-37, voir aussi p. 247). Et pourquoi non "Benard Direx", comme on lit très bien ici comme ailleurs (pp. 158-159) ? Le graveur parisien Robert Bénard (1734-1786) est au moins connu pour avoir dessiné personnellement beaucoup de planches de l'Encyclopédie de d'Alembert et Diderot puis supervisé (direx<it>) dans son atelier les gravures de nombreux autres livres, dont ceux de Cook et de Buffon. Une autre légende à visée historique (p. 45) attribue en partie la dépopulation de l'intérieur de Santo, au cours du xixe siècle, aux "planteurs de bois de santal», etc. Plus d'exactitude s'imposait pour ces traits de l'histoire et de la culture des Ni-Vanuatu auxquels est aussi destiné cet ouvrage.

3 Si certains d'entre eux se trouvent remerciés en fin de volume, le corps du texte, principalement dévolu aux divers chantiers (ou "ateliers ») des naturalistes, à leurs méthodes et à leurs résultats, ne s'intéresse qu'épisodiquement aux autochtones, pour évoquer le plus souvent les réactions qu'ils ont marquées à la présence des scientifiques et trop rarement leurs représentations ou leurs conditions de vie ordinaires. L'ouvrage consacre quelques pages (pp. 148-152) au "climat politique de méfiance» ayant conduit à retirer du projet d'expédition « tout un volet d'enquête ethnologique sur les connaissances naturalistes des populations locales et sur les usages traditionnels de la faune et de la flore» (voir par ailleurs, dans le présent Jso, les articles de Fabienne Tzerikiantz et Elsa Faugère). Quels bénéfices rapides les habitants de Santo pouvaientils attendre de cette coûteuse expédition occidentale? « 15 à 20000 euros » (p. 56) sur " un budget de 1,1 million» (p. 11), peut-être aussi des mesures d'urgence contre certaines espèces invasives (fourmis électriques, lianes envahisseuses, pp. 156-174), des soins médicaux ou des débuts de formation dispensés çà et là, rien que de très ponctuel en tout cas, du fait de la volonté des organisateurs du projet d'éviter qu'il «soit confondu avec un projet de développement rural ou humanitaire, [...] deux types d'opérations différentes qui relèvent de métiers et d'acteurs différents » (p. 56).

Déplorant d'avoir eu à combattre, sans grand succès à Santo, « un véritable fantasme sur les retours économiques de ces inventaires biologiques» et l'éventuelle exploitation par les Occidentaux des patrimoines biologiques autochtones, diffusé d'après lui par "certains responsables d'action de développement et anthropologues " auprès des populations locales (pp. 150-151), Philippe Bouchet ne dissimule pas non plus, dans la postface qu'il signe pour le MNHN avec les co-organisateurs de l'expédition (Hervé Le Guyader pour l'IRD, Olivier Pascal pour Pro-Natura), que les « retours scientifiques » de ces travaux sont eux-mêmes loin d'être garantis, y compris dans les pays dits riches, du fait du manque de moyens humains, matériels et financiers. Ce dernier texte, "Surmonter le "handicap taxonomique" ", ne se limite pas à formuler des craintes pour les délais d'étude des échantillons collectés lors de l'expédition Santo 2006, il précise les objectifs modestes mais raisonnables de ce type d'inventaire. La perspective "linnéenne " d'un catalogue exhaustif de toutes les formes de vie de la planète, hors d'atteinte quelques moyens qu'on y mette, relève elle aussi du fantasme, parfois entretenu auprès du grand public à propos d'expéditions analogues. Mais telle qu'elle fut pratiquée à Santo, l'exploration même ponctuelle de la biodiversité, surtout à l'échelle microscopique où elle est la plus riche, est indispensable aux grandes 
opérations de conservation de la nature, qui n'envisagent généralement que le vivant visible à l'œil nu, et parfois même le plus spectaculaire. Cette défense et illustration de l'exploration de la «biodiversité cryptique » donne tout son sens à l'ouvrage et aux investigations parfois absconses qu'il relate, et il était en somme nécessaire que ce livre "décrypte » auprès du " grand public » les motifs nobles et légitimes de l'expédition Santo 2006. 